\title{
Effects of urban underlying surface on fluid motion in the atmospheric boundary layer
}

\author{
Zhonghua Gao \\ School of Electric Power Engineering, North China Electric Power University, Baoding 071000, \\ China.
}

1194892568@qq.com

Keywords: Atmospheric boundary layer, urban underlying surface, $k-\varepsilon$ turbulence model, numerical simulation

\begin{abstract}
Atmospheric boundary layer is the conveying channel of the earth's atmospheric momentum, heat, and all kinds of material. The main characteristic of the fluid motion in the atmospheric boundary layer is the turbulence, and the wind speed of atmospheric boundary layer increases with the increase of height, wind direction and temperature will change with the height also. Since the atmospheric boundary layer is in the rotation of the earth, when the atmosphere flow on the surface, various flow properties are affected by underlying surface.

In this paper, regard the effects of urban underlying surface on fluid motion in the atmospheric boundary layer as the research direction. In order to analyze the effects of individual buildings and urban buildings cluster on the atmospheric boundary layer of fluid motion and make a comparative analysis of the two, the numerical simulation of the equilibrium atmosphere boundary layer based on the standard k- $\varepsilon$ turbulence model is carried out by FLUENT software.
\end{abstract}

\section{Introduction}

With the accelerated development of urbanization, the urban buildings are tend to be more intensive and gradually increasing in height. The thermal and dynamic processes of urban surface layer have effect on the fluid motion in the atmospheric boundary layer of the urban regional, thus affecting the urban regional climate environment. The main characteristic of the fluid motion in the atmospheric boundary layer is the turbulence, and it's the results of the effects of underlying surface. Different underlying surface has a significant impact on the fluid motion of the atmospheric boundary layer, its radiation properties, heat capacity, water content, roughness and so on are not the same.

The paper focuses on the influence of the urban underlying surface on the fluid motion in the atmospheric boundary layer. To simulate the influence of urban buildings on the fluid movement in the atmospheric boundary layer, the changes of wind speed, temperature and pressure are simulated by FLUENT.

\section{Turbulence Model}

\subsection{Self -Sustaining of equilibrium atmospheric boundary layer}

The research direction of this paper is the distribution of wind load on the building structure of the atmospheric boundary layer with the urban buildings as the underlying surface. In order to obtain the distribution of wind load on the building, it is necessary to deal with the atmospheric boundary layer reasonable. And the equilibrium atmospheric boundary layer is required to meet some suitable boundary conditions: self balanced or self-sustainable inflow conditions, that is, various properties of the flow field (velocity, turbulence, etc.) does not change with the flow direction. However, its difficult to achieve the self-sustaining equilibrium of inflow boundary conditions for atmosphere boundary layers in the simulation. 


\subsection{Standard k- $\varepsilon$ turbulence model}

Focusing on the problem, the paper choose the the equilibrium atmosphere boundary layer which based on the standard k- $\varepsilon$ turbulence model to simulate. According to the inlet parameters obtained by experimental results and the corresponding modified wall function model to make self hold simulation of boundary layer. First, to set the boundary conditions:

The non equilibrium wall function is used for the flow near the wall surface. And the selected fluid is air, the kinematic viscosity is Sutherland, the initial wind velocity is set to 20, pressure is atmospheric pressure, and the temperature is $300 \mathrm{~K}$.

\section{Numerical Simulation}

\subsection{Effects of individual buildings on the atmospheric boundary layer}

Firstly, the individual buildings is chosen as the urban underlying surface, then the effect of the individual buildings on fluid motion of the atmospheric boundary layer is simulated.

a. Division of the grid of the tall individual buildings

To simplify the building into a cube model, and draw a two-dimensional grid. The 2D mesh is the longitudinal section of the building model, which is the result of the wind flow around the tall individual building.

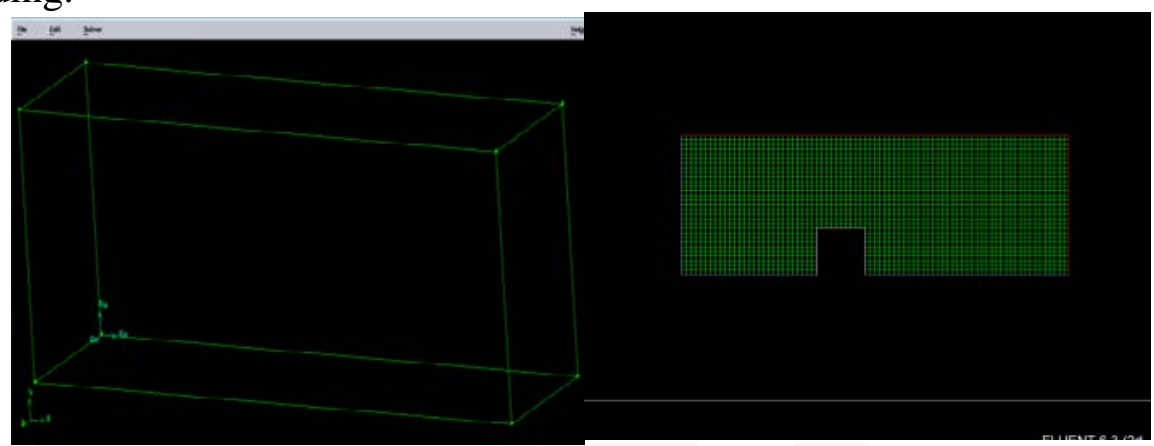

Fig.1 The building model

Fig.2 The two-dimensional grid

b. Velocity profile

The flow speed of wind will change in the atmospheric boundary layer around the tall individual building. Based on the initial boundary conditions above, the velocity distribution is obtained as follows:

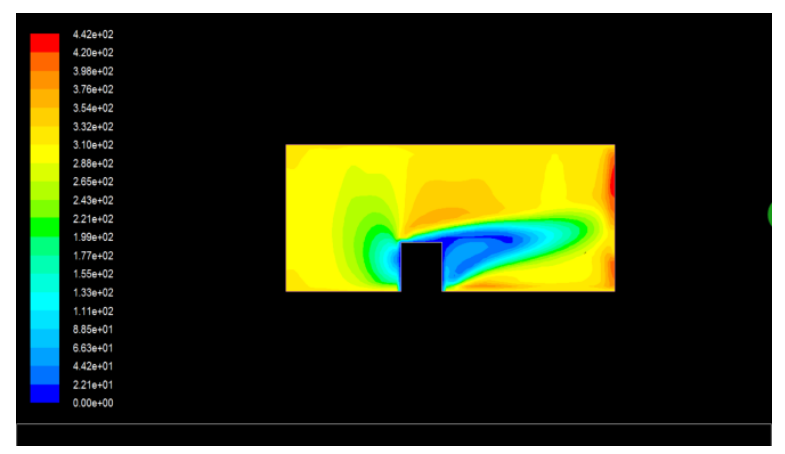

Fig.3 The distribution map of wind speed

It can be seen from fig.3 that the wind speed increases with the increase of height, and it has certain characteristics of turbulence. And at the right side of the building, there is a tail vortex area. c. Pressure profile 


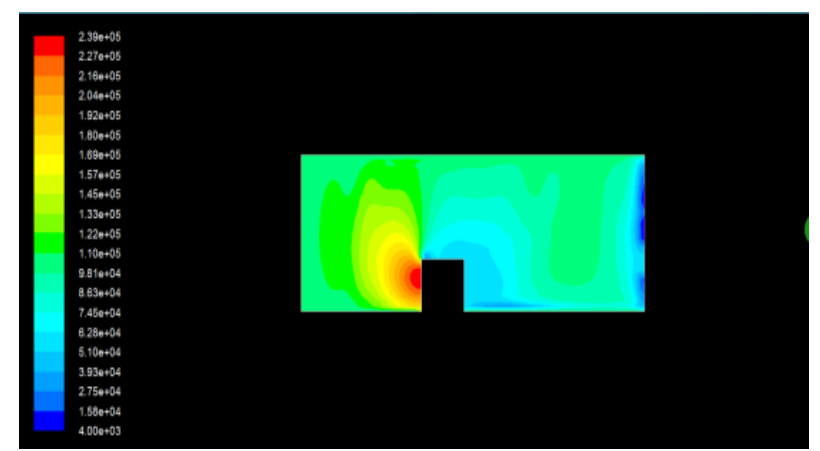

Fig.4 The distribution map of pressure

The simulation of pressure show that the pressure increases with the height, and showed a decreasing trend. And the high-rise on the windward side of the positive pressure and the leeward side of the negative pressure exists huge pressure difference, which caused the strong pressure gradient force, to accelerate the air flow.

d. Temperature profile

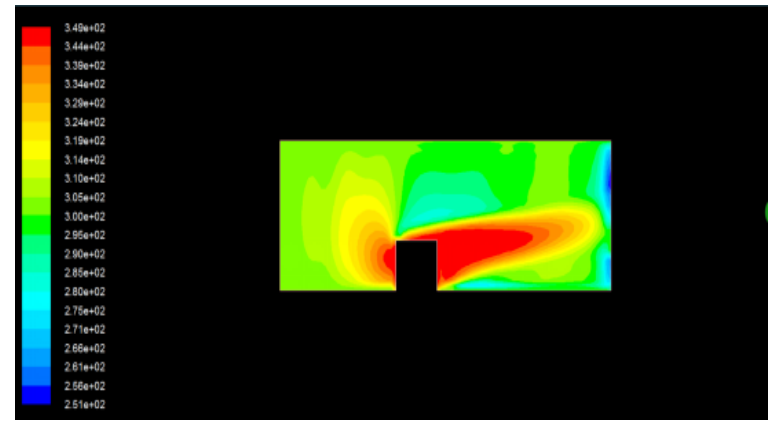

Fig.5 The distribution map of temperature

The atmospheric temperature varies with altitude, and the rate of change has a direct impact on the stability of the atmosphere.

\subsection{Effects of urban buildings cluster on the atmospheric boundary layer}

On the basis of the individual buildings, the urban buildings cluster are chosen as the urban underlying surface.

a. Division of the grid of the urban buildings cluster

First, make the two-dimensional mesh of the urban buildings cluster:

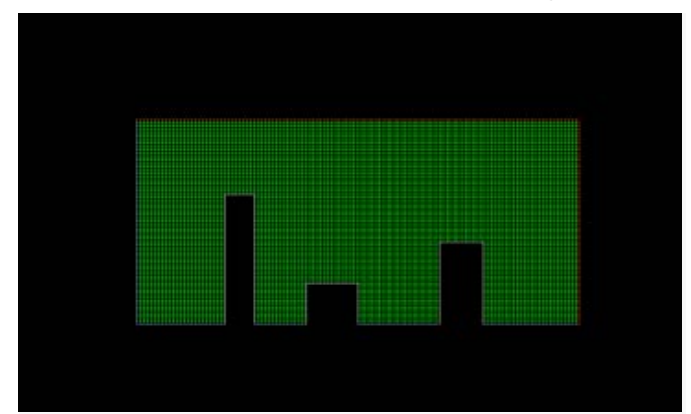

Fig.6 The two-dimensional grid 
b. The profile of wind speed, pressure, temperature
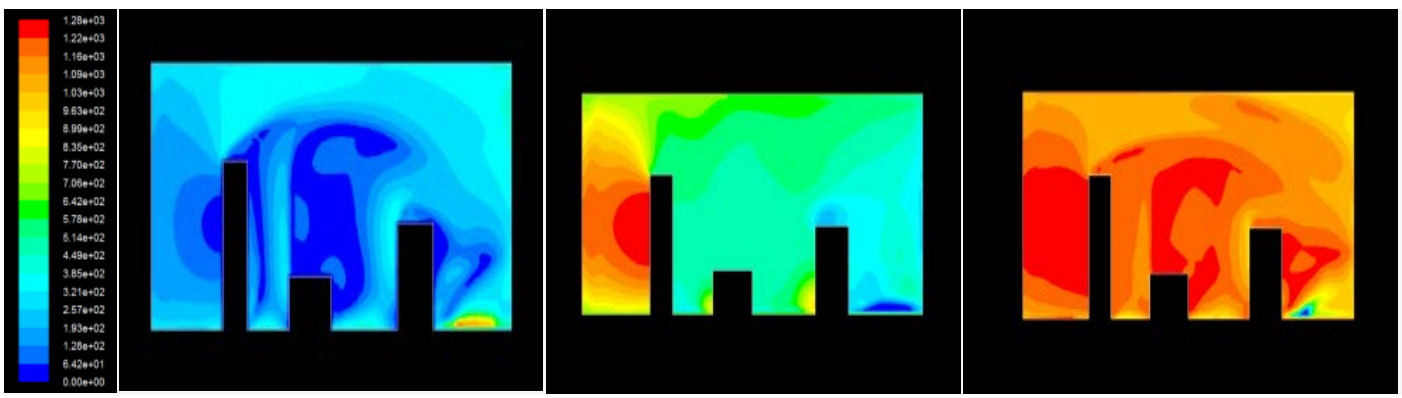

Fig.7 The distribution map of wind speed, pressure,temperature

Due to the existence of urban buildings cluster, increasing the surface roughness, and the average height of the urban canopy layer, thereby reducing the ventilation capacity of the city. And the wind speed of Low-rise buildings are significantly reduced. In general, to improve urban ventilation capacity, the tall buildings have a certain advantage.

\section{Summary}

(1) Overall Characteristics:

Within the atmospheric boundary layer, the wind speed increases with the increase of height, was influenced by surface roughness produced by frictions. The reduce of wind energy lead to the decreases of wind speed, and the degree of reduction increases with the height above the ground.

(2) wind around the building features:

The corner of the building has sharp corners edges, wind flow showed blunt shape, resulting in local disturbances. The flow field around the building is divided into the displacement zone, separation zone, the cavity area, wake zone.

The flow and the building meet in front of the building to form a displacement area, in the front and the lower direction of flow produced maneuver. After the top of the building and on both sides of the corner, the gas produce the flow separation phenomenon, then form a separation zone. At the back of the building, the cavity is formed, and the air flow rate is low. Subsequently, due to the loss of kinetic energy of the flow of the wake area, generally extended to several times the height of the building in the distance. Depending on the Reynolds number, the wake flow will be different from the degree of turbulence.

\section{References}

[1]FLUENT Inc.Modeling Turbulence:Chapter 11.FLUENT6.2 User’s Guide. . 2005

[2]Blocken B, Stathopoulos T, Carmeliet J.CFD simulation of the atmospheric boundary layer-wall function problems. Atmospheric Environment . 2007

[3]HARGREAVES D M,WRIGHT N G.On the use of the k- $\varepsilon$ model in commercial CFD software to model the neutral atmospheric boundary layer.

[4]ZHANG Boyin SANG Jianguo WU Guochang State Key Laboratory for Turbulence and Complex System, Peking University, Beijing 100871, China 